\title{
LA UNIVERSITAT OBERTA DE CATALUNYA (UOC): INNOVACIÓN EDUCATIVA Y TECNOLÓGICA EN EDUCACIÓN SUPERIOR
}

\author{
(THE OPEN UNIVERSITY OF CATALONIA (UOC): EDUCATIONAL AND TECHNOLOGICAL \\ INNOVATION IN HIGH EDUCATION)
}

\author{
Josep M. Duart \\ Lourdes Salomón \\ Pablo Lara \\ Universitat Oberta de Catalunya (España)
}

\section{RESUMEN}

La Universidad Oberta de Catalunya (UOC), creada en 1995 por iniciativa del Gobierno de Cataluña, nace con la finalidad de facilitar la formación de las personas a lo largo de la vida. Como institución creada en la Sociedad de la Información para la Sociedad de la Información se dota de una estructura flexible y fundamenta su modelo educativo en la innovación y en el uso intensivo de las TIC. A partir del Campus Virtual de la UOC, pionero en educación superior, estudiantes y profesores interactúan asíncronamente con la finalidad de facilitar un proceso de enseñanza y aprendizaje que procure la consecución de los objetivos propuestos. El presente artículo muestra la evolución de la UOC en sus once años de historia y plantea los retos de la institución en el momento actual.

Palabras clave: Universitat Oberta de Catalunya, UOC, e-learning, campus virtual, universidad, modelo educativo, innovación.

\begin{abstract}
The Open University of Catalonia (Universidad Oberta de Catalunya, UOC), created in 1995 by initiative of the Government of Catalonia, was born with the purpose to facilitate people's long life formation. As an institution created in the Information and Communication Society, is endowed of a flexible structure and supports its educational model in the innovation and in the intensive use of the
\end{abstract}


ICT. From the Virtual Campus of the UOC, pioneer in higher education, students and professors interact with the purpose to facilitate a process of teaching and learning that wants to reach the objectives proposed. The present article shows the evolution of the UOC in its eleven years of history and strikes the challenges of the institution at the present moment.

Key words: UOC, Open University of Catalonia, e-learning, virtual campus, university, higher education, innovation, educational model.

"La UOC -Universitat Oberta de Catalunya- es una institución que surge de la sociedad del conocimiento con la misión de facilitar la formación de las personas a

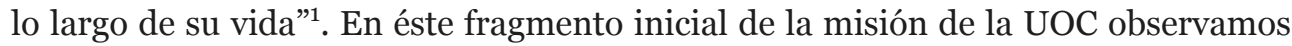
ya su objetivo fundacional: la formación de las personas a lo largo de la vida en el contexto de la sociedad del conocimiento y desde una institución fruto de la sociedad del conocimiento. Desde su inicio la UOC envía un mensaje claro como institución: la voluntad de ser una institución educativa capaz de satisfacer las necesidades educativas de las personas a lo largo de la vida en una sociedad informacional en constante cambio. Para ello, universidad en sí misma, en tanto que institución educativa, debe ser un ejemplo de modelo de organización de educación superior en la sociedad del conocimiento y debe ser capaz de releer y adaptar los fines sociales de la universidad ante los retos y oportunidades de la Sociedad de la Información y las demandas y necesidades de sus ciudadanos.

La innovación continua debe ser una capacidad básica para cualquier institución en la Sociedad del Conocimiento. Sin innovación, sin capacidad de adaptación constante, gestión del cambio y mejora continuada es difícil sobrevivir como institución en nuestra sociedad actual. Las personas que formamos esta sociedad demandamos a nuestras instituciones, y especialmente a las educativas, respuestas y propuestas claras que nos ayuden a mantener nuestra capacidad de mejora constante en esta sociedad. La misión básica de la universidad de formar ciudadanos, de crear y de difundir el conocimiento la convierte en una de las instituciones clave de la sociedad del conocimiento. La apuesta por la formación de calidad, por la innovación educativa, por la investigación básica y aplicada en las necesidades sociales, por la difusión y accesibilidad al conocimiento, etc, no es sólo ya una respuesta actual a la misión de la universidad, es una demanda de los gobiernos y de las administraciones educativas de todos los países fundamentada en la necesidad de mantener la cultura, la competitividad, la creatividad y el espíritu emprendedor de sus ciudadanos. 
La UOC es el resultado del empuje de una sociedad concreta por dotarse de una universidad de nueva generación capaz de dar respuesta a la formación continua de sus ciudadanos. El Gobierno de Cataluña promueve la creación de la UOC y concreta en sus normas la manifestación de la voluntad institucional de "integración en el sistema universitario de Cataluña con la finalidad de prestar servicio a la cultura catalana y proyectarla en el mundo" 2 . La UOC parte, por tanto, de una realidad cultural y de una identidad concreta, la catalana, para desde ella proyectarse como una institución global, internacionalizada y multilingüe (Duart; Kiselyova, 2003). De lo local a lo global, este es el proceso constante bidireccional en la mayoría de las instituciones actuales.

Para conseguir esos los retos que hemos expuesto, en sus normas de funcionamiento iniciales, en el año 1995, la UOC se propone los siguientes objetivos:

- El impulso, la mejora y la innovación en la docencia y el aprendizaje a lo largo de la vida por medios no presenciales.

- El fomento y el desarrollo de la investigación científica en todos los campos del conocimiento en los que esté presente la universidad, especialmente, en el estudio de la sociedad del conocimiento.

- La difusión del conocimiento, la transferencia de tecnología, saber hacer e innovación en los campos de la formación y de la cultura asociados al uso intensivo de las tecnologías de la información y de la comunicación (TIC).

La UOC se construye, por tanto, basada en los pilares de la innovación educativa en educación no presencial, en el fomento de la investigación científica de la sociedad de la información y con una clara voluntad de difusión del conocimiento.

\section{LA CREACIÓN DE LA UOC: SU ENTORNO Y SU MISIÓN FUNDACIONAL}

\section{El sistema universitario catalán a partir de los años 90}

A principios de los años 90 el Gobierno de Cataluña impulsó la creación de nuevas universidades - inicialmente a partir de la segregación de los campus que la Universidad de Barcelona tenía fuera de la ciudad- que debían caracterizarse por convertirse en instituciones educativas superiores enraizadas en su territorio. La finalidad era descentralizar y dinamizar el sistema universitario así como fomentar el desarrollo económico y cultural en todo el territorio catalán a través 
de la implantación de centros de formación e investigación superior. Las nuevas universidades de titularidad pública que se crearon en aquellos años, fruto de esta decisión política, fueron: en 1990 la Universidad Pompeu Fabra en Barcelona, en 1991 las Universidades de Girona, de Lleida, y la Universidad Rovira i Virgili en Tarragona. Así mismo se creó en 1991 la Universitat Ramon Llull, de titularidad privada, como resultado de la agregación de diferentes instituciones de educación superior, ya existentes, dependientes de diferentes órdenes religiosas o diócesis catalanas ${ }^{3}$.

El año 1995 el Parlamento de Cataluña aprueba por unanimidad la creación de la Universitat Oberta de Catalunya ${ }^{4}$, como una fundación privada sin afán de lucro, participada por la administración pública, y bajo la modalidad de universidad a distancia. Posteriormente se crearon las universidades privadas de Vic, la Internacional de Catalunya y la Abat Oliva, que juntamente con las públicas ya existentes antes de los 90 (Universidad de Barcelona, Universidad Autónoma de Catalunya y Universidad Politécnica de Catalunya) forman el actual sistema universitario catalán.

Las universidades han actuado como motores del desarrollo económico, demográfico, tecnológico, urbanístico del territorio catalán y así como también de la mejora constante y la innovación educativa, cultural y social en los territorios en los que se han asentado. Más de una década después existe en Cataluña un consenso evidente entre los principales actores sociales sobre el papel relevante que desempeñan los centros de educación superior en sus respectivos territorios, convirtiéndose en motores del desarrollo económico y social del entorno en relación a la formación del capital humano para el fomento de la cultura y del conocimiento. A esta nueva misión que deben cumplir las universidades estrechamente vinculadas al territorio se la ha llamado Tercera misión ${ }^{5}$.

La realidad de las universidades creadas en la década de los 90 corresponde a la descripción de centros de dimensiones más reducidas que las universidades tradicionales y con menores recursos humanos y económicos para la investigación. Debido a ello han actuado estratégicamente buscando la excelencia en la docencia y para ello han investigado sobre la aplicación de nuevos métodos docentes y han concentrado sus esfuerzos de investigación, de forma especial, en áreas concretas en conexión con los intereses del entorno más inmediato y a los que se han destinado todos los recursos humanos y económicos. Se trata de universidades con estructuras más ágiles y dinámicas que los centros universitarios tradicionales y son éstas precisamente las que están liderando el proceso de adaptación de las universidades al nuevo Espacio Europeo de Educación Superior. 


\section{La especificidad de la UOC en el sistema universitario catalán y español}

La creación de la UOC en el año 1995 es un caso significativo de la política universitaria catalana de los años 90. La UOC se crea como una universidad a distancia con una vocación clara de acercar el conocimiento a todas las capas de la sociedad y haciendo de este modo factible una distribución más equitativa del conocimiento en sintonía con las declaraciones de la UNESCO y las declaraciones de derechos humanos. Para ello, y de forma pionera, el gobierno y el equipo fundacional de la UOC apuestan por un modelo innovador, basado en la comunicación asíncrona en las relaciones docentes e institucionales, y soportado tecnológicamente en Internet. Nacía de esta forma la primera universidad a distancia de nueva generación, lo que posteriormente conocimos como universidad virtual o universidad online (Tiffin; Rajasingham, 2003).

En el panorama universitario español, la Universitat Oberta de Catalunya se ha posicionado como una universidad innovadora y pionera en el uso de las nuevas tecnologías aplicadas a la docencia universitaria. Sin embargo la auténtica innovación de la UOC está precisamente en su metodología docente en la que la evaluación continua (Dochy; Segers; Dierick, 2003) del aprendizaje se ha convertido en el auténtico motor del proceso de aprendizaje ${ }^{6}$. Un modelo pedagógico basado en la formación a través de la red utilizada para facilitar la comunicación entre profesor, estudiantes y recursos mediante herramientas asíncronas de comunicación telemáticas (Cabero, 2006).

\section{La diversidad lingüística de la UOC}

En las universidades catalanas se produce un fenómeno lingüístico diferente al del resto del estado español 7 , la coexistencia de dos idiomas oficiales en las aulas, el castellano y el catalán. Si bien el idioma vehicular de las universidades es el catalán por ser la lengua oficial, ambas lenguas conviven con total normalidad en las universidades catalanas.

La UOC en sus inicios creo únicamente un campus virtual en lengua catalana, pero tras 5 años de funcionamiento en esta lengua y dando servicio a la comunidad lingüística catalana, en el curso 2000-2001 creó un nuevo campus virtual en español, configurándose de este modo dos campus diferentes por razones de lengua, pero con idéntica metodología e iguales servicios académicos. Los materiales docentes, las actividades de evaluación continua, los exámenes, los recursos didácticos, etc. son idénticos con independencia de la lengua en la que se imparta la docencia. 
La experiencia que ha adquirido la institución al gestionar toda la información académica en dos lenguas simultáneamente, le ha permitido plantearse la posibilidad de impartir su docencia en otras lenguas como el inglés o el francés, a la vez que está reflexionando sobre la posibilidad de crear aulas plurilingües con la finalidad de ofrecer una educación que sea reflejo de toda la riqueza cultural europea. (Delgado; Oliver; Salomón, 2006).

La preocupación por los aspectos lingüísticos se traduce también en la investigación que se está llevando a cabo en la UOC. Algunos ejemplos de esta línea de investigación es un proyecto llamado @languages: Virtual Learning Languages in a Multicultural Professional Environment que se enmarca dentro del programa de la Comunidad Europea Leonardo da Vinci. Su objetivo es realizar un producto innovador y de gran calidad para el aprendizaje de las lenguas usando las nuevas tecnologías. Así como, el proyecto Detecting and Removing Obstacles to Foreign Languages Teaching Abroad (Drofolta) que lidera la UOC dentro del Plan de acción sobre aprendizaje de lenguas y diversidad lingüística de la Comisión Europea.

Este, es uno de los varios ejemplos de como la institución convierte las especificidades de su actividad docente en objeto de la investigación de sus profesores, creando de este modo redes de investigación sobre su peculiar identidad académica. Esta estrategia de estrecha vinculación entre docencia e investigación permite conseguir la excelencia en la metodología docente y una investigación aplicada en la propia institución que a su vez retroalimenta el círculo virtuoso entre docencia e investigación ${ }^{8}$.

Recientemente la Rectora de la UOC, Dra. Imma Tubella, ha creado un nuevo vicerrectorado en Multilingüismo con la finalidad de impulsar el uso de diferentes lenguas aparte del español, del catalán y del inglés. Existen algunos proyectos concretos de relación con universidades del norte de África que comportan la habilitación de un Campus Virtual y materiales de aprendizaje en lengua árabe.

\section{La UOC en el contexto europeo de educación superior}

La UOC, al igual que el resto de universidades europeas, está inmersa en un proceso de cambio importante con el fin de conseguir la adaptación a los objetivos que propone el Espacio Europeo de Educación Superior (EEES). Las universidades europeas de más reciente creación, como la UOC, aportan experiencias muy próximas a los objetivos que la Declaración de Bolonia ${ }^{9}$ persigue. En el cumplimiento de estos objetivos, las tecnologías de la información y la comunicación (TIC) juegan un papel fundamental. 
El EEES pretende que el estudiante adquiera a lo largo de su formación unas determinadas competencias que lo preparen para la vida profesional, lo que exige un renovado diseño curricular y un material docente adecuado a la consecución de dichos objetivos. La estructuración de los curricula de acuerdo con las competencias que exigen el mundo profesional, pretende dotar a los estudiantes, no únicamente de los conocimientos de tipo teórico de las enseñanzas tradicionales, sino que además pretende que los estudiantes adquieran todas aquellas habilidades, destrezas, procedimientos, técnicas, etc, que les serán exigidas para desarrollar con éxito su profesión en la vida laboral.

Las estrategias para el establecimiento de nuevas relaciones en el contexto europeo pueden llevarse a cabo desde su vertiente investigadora o desde su ámbito docente. En el primero la universidad apostó por la creación del Internet Interdisciplinary Institute, (IN3), ${ }^{10}$ como centro de investigación base de la UOC. El IN3 acoge los diferentes grupos de investigación de la UOC y la coordinación de numerosos proyectos europeos e internacionales en los que los profesores e investigadores de la UOC participan. El IN3 colabora, a su vez, en la política estratégica de adaptación al EEES, en lo que se refiere a la investigación, y promueve alianzas europeas a partir del Programa de Doctorado en Sociedad de la Información o bien directamente con otros institutos de investigación de ámbitos de conocimiento similar como el Oxford Internet Insitute.

En el ámbito docente el profesorado de la UOC se convierte en experto en metodologías de formación virtual, debido a su práctica cotidiana en la creación e impartición de asignaturas con uso intensivo de las TIC. A su vez se convierten en referentes en su ámbito de especialidad para este tipo de docencia. La estrategia de internacionalización en el marco europeo tiene en cuenta el potencial del profesorado propio y colaborador de la UOC en el establecimiento de alianzas académicas, de cooperación entre profesores con la finalidad de generar proyectos de investigación en el marco de las convocatorias europeas o alianzas para el desarrollo de programas académicos conjuntos.

Desde su inicio la UOC ha apostado por participar en diferentes redes europeas de educación a distancia o de educación y uso de las TIC. En este sentido es miembro de EDEN (European Distance Educaction Network), de EADTU (European Asociation of Distance Teaching Universities), EAIR (European Association for Institucional Research in Higher Education) así como otras de ámbito global como EDUCAUSE, el OBHE (Observatory of Borderless Higher Education). La participación en estas y otras organizaciones, así como la promoción del intercambio de estudiantes y profesores en entornos de docencia virtual y la creación de cursos compartidos permite a estudiantes y profesores desarrollar las competencias lingüísticas, 
comunicativas y culturales propias de la diversidad de la sociedad europea de hoy. Esta es una clara apuesta de introducir la UOC dentro de los hogares de muchos estudiantes y profesores europeos.

\section{UN MODELO EDUCATIVO INTEGRADO Y FLEXIBLE}

\section{Modelo educativo, interacción y uso de la tecnología}

Desde el punto de vista comunicativo la principal característica del modelo educativo de la UOC es que se basa en la asincronía, es decir, en la no coincidencia ni espacial ni temporal entre los agentes que intervienen en el proceso de enseñanzaaprendizaje. Esto es posible gracias al uso de las tecnologías de la información y de la comunicación (TIC), especialmente internet.

La apuesta por la asincronía no viene definida por la tecnología. Se trata de una apuesta estratégica para facilitar la misión de la institución de formar personas a lo largo de la vida. Estas personas, a las que nos referimos, son adultas y trabajadoras. La media de edad de los estudiantes de la UOC es de 35 a 45 años, trabaja, posee ya un título universitario en el $48 \%$ de los casos y viven, mayoritariamente, en grandes zonas urbanas en las que existen varias universidades presenciales. Este perfil de estudiante necesita un sistema de acceso a la formación flexible y adecuado a sus ritmos temporales de trabajo y que facilite la conciliación de la vida profesional con la personal y/o familiar. De ahí que la UOC haya optado, de forma coherente con su misión fundacional, por un sistema asíncrono.

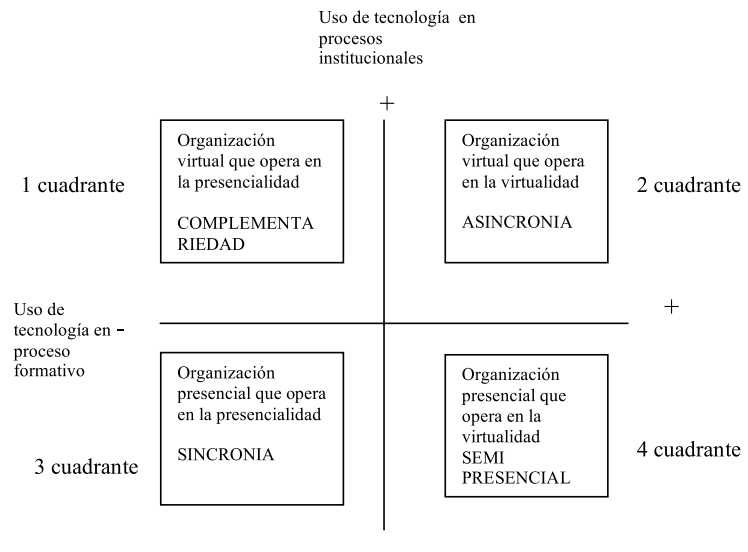

Figura 1. Topología de universidades según uso de la red 
Pero a pesar de la modalidad asíncrona y de la flexibilidad que permiten las tecnologías, la UOC no optó en el inicio por un sistema realmente abierto al estilo de las clásicas open universities. Los estudiantes no pueden empezar el curso cuando quieren o seguir el curso completamente a su ritmo. Se sacrificó esta flexibilidad para poder ofrecer a los alumnos un modelo más interactivo, basado en la comunicación con los profesores y los estudiantes y en el que el aprendizaje cooperativo fuese posible. Para ello se necesita de la existencia de grupo-clase, de un aula, y de ahí que se inicien las actividades académicas -especialmente en el primer y segundo ciclo de formación- dos veces al año, como en la mayoría de las universidades presenciales.

El modelo educativo de la UOC es un modelo centrado en el estudiante (Duart; Sangrá, 2000). Más allá del significado pedagógico de la centralidad en el discente, lo que se valora en el caso de la UOC es que la organización del proceso de enseñanza y aprendizaje gira alrededor del estudiante. No estamos haciendo referencia a teorías de aprendizaje, sino a la didáctica y organización del proceso formativo. En los modelos de formación de educación a distancia caben diferentes apuestas de centralidad. Encontramos modelos centrados en los contenidos de aprendizaje, es decir, en la creación y distribución de materiales de aprendizaje que favorecen un sistema de autoaprendizaje por parte de los estudiantes. Otros modelos se centran más en el profesorado y su rol como seleccionador, distribuidor y dinamizador de los contenidos de aprendizaje. Ambos modelos son adecuados y practicados por diferentes universidades a distancia en el mundo. En ambos casos también la tecnología juega un papel determinante, ya sea como herramienta de distribución de la información en los modelos centrados en los contenidos de aprendizaje, o como sistema de comunicación síncrona (videoconferencia, por ejemplo) en los modelos centrados en el profesorado. Cada modelo se dirige a un determinado perfil de estudiante y tiene metodologías y didácticas diferenciadas.

La centralidad en el estudiante que propone la UOC, basada en la asincronía que proporciona el uso de Internet y de su potencial comunicativo y de acceso a la información, configura un modelo organizativo y tecnológico específico diseñado para dar respuesta a esta modalidad formativa. En cierta forma el modelo de la UOC tiende también a la centralidad en el profesorado, ya que ejerce un papel destacado en el proceso formativo como facilitador del proceso de aprendizaje, pero tiende menos a la centralidad en los contenidos de aprendizaje, ya que a pesar que estos existen y son elaborados en formato didáctico por la institución están siempre complementados por otros materiales presentados por los profesores, así como por las actividades de evolución y los comentarios de los profesores y de los alumnos en el aula. De ahí también la importancia de la interacción comunicativa. 


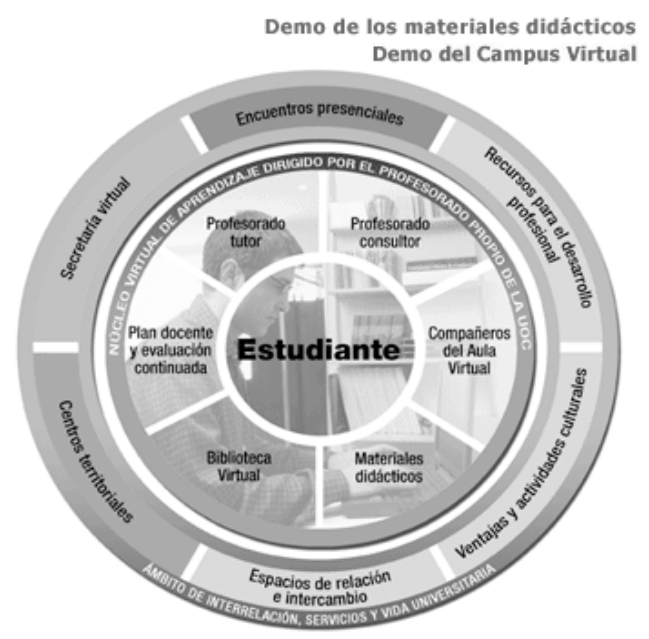

Figura 2. Modelo educativo de la UOC

El elemento que, a nuestro entender, expresa la centralidad organizativa y metodológica del modelo de la UOC es el plan docente o guía de aprendizaje. Se trata de un documento redactado por el profesorado de la universidad que expone al estudiante la organización del proceso de enseñaza y aprendizaje desde el primer día de clase. Es la guía base a partir de la cuál se accede a los objetivos de aprendizaje, a los contenidos, a los materiales de aprendizaje, a las actividades, al sistema evaluativo, bibliografía, planificación, etc. Es un documento de referencia para el estudiante que, en cierta forma, colabora en fomentar su proceso de autogestión del aprendizaje.

Basándonos en esa centralidad del estudiante en el proceso de enseñanza y de aprendizaje, el modelo educativo se articula a partir de los siguientes principios:

- Flexibilidad

Entendemos la flexibilidad desde el punto de vista de la organización del estudiante de su proceso de aprendizaje. Se trata de una dinámica de autogestión educativa. Al conocer el estudiante desde el primer día la planificación del trabajo a través del documento del plan docente se le da la posibilidad, pactada con el profesor, de elegir su ritmo de trabajo. Si bien se le aconseja seguir la evaluación continuada, el estudiante puede decidir optar por ir directamente a una prueba final, por ejemplo. La asincronía es una muestra más de la apuesta por la flexibilidad de cara al estudiante ya que le permite una interacción adecuada a su ritmo personal de vida. 
- Personalización

Los planes de estudio y los currículos de las asignaturas pretenden adaptarse a las necesidades de los estudiantes, con la finalidad de personalizar su proceso de formación. Se apuesta también, por ejemplo, al reconocimiento de la formación previa o de los conocimientos adquiridos en los procesos laborales. Este es un elemento clave teniendo en cuenta que la UOC se dirige especialmente con estudiantes adultos. La personalización en el proceso de aprendizaje permite una cierta adecuación de los contenidos y de las actividades del aula a los perfiles profesionales o intereses formativos de los estudiantes.

- Interacción

La interacción se pretende multidireccional. Básicamente se fundamenta en la relación entre estudiante y profesor, pero también se fomenta en cada aula la interacción entre estudiantes y entre estudiantes y profesores con los contenidos de aprendizaje. La interacción es clave en el proceso formativo de la UOC, de ahí que como hemos mencionado anteriormente no nos encontramos ante un modelo de autoaprendizaje, sino en un modelo formativo en el que la comunicación interpersonal y su papel en el proceso juega un papel clave. La interacción se entiende como una oportunidad para el aprendizaje, no se ofrece únicamente como elemento de apoyo a los contenidos de aprendizaje.

- Cooperación

Se trata de uno de los elementos más destacados en el modelo educativo de la UOC. Se fomenta la cooperación como estrategia de aprendizaje y de construcción de conocimiento a partir de las actividades que se detallan en los planes docentes. Este principio estimula, a la vez, el uso de las TIC como instrumento de construcción compartida de conocimiento. Las actividades cooperativas entre estudiantes se realizan de forma asíncrona, y cada vez más se incrementan con el uso del potencial que ofrecen las nuevas plataformas tecnológicas de aprendizaje.

\section{El sistema docente de la UOC: innovación en la organización del proceso de enseñanza no presencial}

Desde su inicio en 1995 la UOC se dota de un sistema de organización académica innovador, basado en la cooperación y las alianzas con todas las universidades del sistema universitario catalán. Esta decisión, fruto del consenso inicial propiciado 
por la administración educativa y las universidades del sistema catalán, facilita la configuración de un sistema docente basado en diferentes figuras académicas, algunas con plena dedicación en la UOC y otras que colaboran puntualmente. Se constituye, por tanto, un sistema organizativo de la docencia flexible que se basa en la cooperación interuniversitaria.

La UOC organiza la docencia a partir de cuatro figuras claves: el profesor propio (a tiempo completo en la UOC), el autor de los contenidos (por encargo), el consultor (profesor de otra universidad o profesional liberal contratado a tiempo parcial) y el tutor (profesor o pedagogo contratado a tiempo parcial). De esta forma se consigue una estructura docente propia de la UOC bastante reducida, mientras que la estructura contratada de forma parcial, según las necesidades de matricula, es bastante más amplia. Esto configura una estructura organizativa flexible e innovadora. Exponemos a continuación una breve explicación de cada una de las figuras docentes.

- El profesor de la UOC

Es un académico de la disciplina que se agrupa con otros colegas en un Estudio o Facultad. Se dedica a tiempo completo a la UOC y tiene la máxima responsabilidad en la tarea docente y académica. En otras palabras, es quien determina -juntamente con sus otros colegas profesores- el plan de estudios de una carrera, decide el currículo de las asignaturas, selecciona los equipos de profesores que redactaran los materiales de aprendizaje, supervisa la acción de los profesores (consultores) a tiempo parcial, da el visto bueno a los exámenes, etc. El profesor de la UOC es quien ostenta la libertad de cátedra del sistema docente. Además, claro está, como profesor es también investigador y debe participar en proyectos de investigación.

El profesor de la UOC no imparte la docencia directamente, sino que su misión es diseñar la asignatura, crear un equipo docente e implementar la metodología docente en esa asignatura.

- El autor de los materiales de aprendizaje

El profesor de la UOC, una vez decidido el enfoque académico y el esquema de contenidos de una asignatura, procede a decidir y contratar a un autor o equipo de autores para la elaboración de los materiales. El mismo profesor puede ser autor, claro está. La derechos de la autoría que realizan estos profesionales externos (pueden ser profesores de otras universidades o pueden ser profesionales en el mercado laboral) se establecen a partir de un 
contrato entre la UOC y el autor. Normalmente los derechos de uso de los materiales de aprendizaje quedan en propiedad de la UOC. Los materiales se desarrollan en diferentes formatos: papel, web, video, etc. No es necesario que los autores conozcan los mecanismos tecnológicos para desarrollar los materiales en diferentes formatos. La UOC pone a disposición de los autores equipos multidisciplinares de tecnólogos y diseñadores instruccionales que facilitan esa labor una vez entregados los originales por parte de los autores. Además, los materiales no son libros de texto, son materiales didácticos, es decir, texto con anotaciones que facilitan el estudio tales como actividades, fotografías, gráficos, objetivos basados en competencias, glosario de términos, etc. La supervisión y la aprobación final de los materiales es siempre competencia del profesor de la UOC.

- Los consultores

Una vez desarrollados los materiales de aprendizaje por los autores se procede a la preparación del Plan Docente de cada asignatura. Para ello el profesor de la UOC selecciona uno o varios consultores, según el número de aulas existentes, para impartir la docencia. El consultor será la persona que interactuará directamente, a través del Campus Virtual, con los estudiantes. El consultor debe conocer los contenidos, claro está, pero además debe tener suficientes conocimientos prácticos para el desarrollo de la docencia en entornos virtuales de aprendizaje. El Plan Docente, que en el caso de existir más de un consultor por asignatura se desarrolla de forma cooperativa, es único para cada asignatura y es el punto clave a partir del cuál se dispone toda la organización docente. El Plan Docente determinará el uso de los materiales que han elaborado los autores, propondrá las actividades de evaluación continua, explorará nuevas vías de información complementaria para el correcto aprendizaje de la asignatura, y claro está, determinará el sistema de evaluación del aprendizaje.

- El tutor

La figura del tutor es una de las novedades más significativas en el modelo educativo de la UOC. Para evitar la deserción de los estudiantes así como para acompañarlos en su proceso de aprendizaje se diseñó la figura del tutor. Su perfil es el de un académico o profesional del ámbito disciplinario en el que el docente esta inmerso, pero que además dispone de conocimientos de los entornos virtuales de aprendizaje, de acompañamiento de los estudiantes, del mercado laboral y de las salidas profesionales de la carrera. Los tutores están muy cercanos a los estudiantes ya que son la única figura que permanece a 
lo largo de todo el período que el estudiante está en la universidad. Mientras que los estudiantes van a tener tantos consultores como asignaturas, tutores sólo tendrán uno, desde el principio de su paso por la UOC hasta el final.

Esta dinámica de organización del sistema docente determina un funcionamiento que sustenta la convicción pedagógica de la UOC de ofrecer un modelo de aprendizaje basado en la interacción comunicativa entre estudiantes y profesores. Dicho de otra forma, el modelo educativo de la UOC no es un modelo sustentado en el autoaprendizaje, o basado en unos materiales de aprendizaje que pueden trabajarse de forma autónoma sin apoyo del docente. Esto es determinante para entender la estructura docente y organizativa de ésta institución.

Con la creación de esta estructura de profesorado, lo que se ha hecho en definitiva, es fragmentar las tareas que tradicionalmente realiza el profesor universitario. La metodología docente queda en manos del profesor de la UOC que actúa como intermediario entre la institución y el equipo docente. Lo que ha sido siempre la función de un solo profesor, en nuestra metodología se ha dividido en unas determinadas funciones que cumple el profesor, (diseño de la asignatura, coordinación de los consultores y asesoramiento metodológico para la docencia virtual) y en otras que realiza el consultor, entre las que cabe destacar el papel de motivador y dinamizador del proceso de aprendizaje del estudiante.

También se han fragmentado entre los consultores las diversas tareas que realiza tradicionalmente un solo docente. $\mathrm{Al}$ consultor se le exige como requisito básico conocer la materia y estar dispuesto a dominar el uso de los instrumentos tecnológicos que pone a su disposición el Campus Virtual. Pero además, de él se espera una determinada actitud, un compromiso profundo por el proceso de aprendizaje de sus estudiantes. Se valora especialmente su actitud positiva para estimular el interés del estudiante por la materia. La interacción entre el contenido y el estudiante no garantiza por sí sola la adquisición de formas óptimas de significados y sentido. El consultor aporta al estudiante la ayuda que necesita para poder adaptar de manera dinámica, y contextual el contenido a aprender. La interacción entre contenido y estudiante no es solitaria sino que el proceso aparece mediado por el consultor que sigue de manera próxima el proceso de aprendizaje de cada uno de sus estudiantes (Onrubia, 2005).

En nuestro entorno docente virtual es preciso que se dedique especial atención a la creación de un contexto que facilite la interacción entre los tres elementos: el estudiante, los contenidos y el consultor. Esta es una tarea que exige una especial 
dedicación por parte del consultor, por este motivo, aunque pueda parecer paradójico, en los entornos virtuales la función del docente se ve aumentada en relación a la docencia presencial ${ }^{11}$. Las TIC amplifican la presencia del docente en el proceso de aprendizaje del estudiante.

La experiencia de más de diez años de docencia virtual nos ha demostrado claramente que construir conocimiento junto a otros es más efectivo que hacerlo individualmente, de modo que el consultor para conseguir este tipo de aprendizaje debe asumir diferentes roles. Además de organizador del trabajo, y facilitador del desarrollo cognitivo del estudiante, es imprescindible que el consultor de la UOC asuma el papel de socializador del contexto de aprendizaje. En nuestro modelo de aprendizaje la comunicación entre el grupo es fundamental, por lo que el consultor debe actuar de moderador, ayudando a que el grupo se comunique de manera eficiente entre sí, y consiga hacer nacer entre sus estudiantes la sensación de pertenencia a un grupo de aprendizaje con objetivos comunes.

\section{INNOVACIÓN Y COOPERACIÓN EDUCATIVA Y TECNOLÓGICA}

Este modelo docente es posible únicamente si existe un sistema de cooperación en el sistema universitario y una gran implicación con la sociedad por parte de la universidad. Más de la mitad de los consultores y tutores de la UOC son profesores a tiempo completo en otras universidades, especialmente en las catalanas, y el resto son profesionales en activo (abogados, informáticos, empresarios, etc). Más de dos terceras partes de los autores de los materiales educativos de la UOC son profesores y profesionales en activo de la sociedad catalana o española. Para desarrollar este complejo sistema la UOC ha establecido convenios con todas las universidades del sistema catalán y con bastantes del sistema español, en los que se definen los sistemas de colaboración entre ambas instituciones.

Sin duda la importancia de este sistema reside en la flexibilidad que aporta. Tan sólo unos datos: la UOC tiene cerca de 35000 estudiantes en el 2006, 180 profesores y casi 2000 consultores y tutores. La flexibilidad es evidente, pero a la vez también, la dependencia del sistema de colaboración y alianzas es total. De ahí la importancia de establecer un sistema de cooperación basado en la colaboración y el aprendizaje mutuo. Exponemos a continuación algunos de los pilares de este sistema de colaboración interuniversitaria. 


\section{Estudiantes adultos y que trabajan}

La UOC se dirige a un colectivo potencial de estudiantes al que no se dirigen las demás universidades catalanas. El estudiante de la UOC se define por ser un adulto (de 30 a 40 años de media) que trabaja (90 \% de los estudiantes de la UOC trabajan). Los estudiantes de las demás universidades son jóvenes y todavía no han accedido al mercado laboral. Esto sucede especialmente en las carreras de primer ciclo. No así en los postgrados en los que la competencia entre universidades puede ser mayor.

De igual forma en el sistema universitario catalán existe una coordinación entre universidades, ejercida por el Departamento de Universidades del Gobierno Catalán, que establece que las demás universidades no van a impartir carreras de primer y segundo ciclo en modalidad virtual, dejando este modelo para la UOC. Esto facilita también la cooperación interuniversitaria.

\section{La innovación educativa}

El modelo educativo de la UOC esta centrado en el estudiante. Internet permite la comunicación asíncrona, y esto permite la relación bidireccional y multidireccional entre profesor y alumno y entre todos los miembros de la comunidad universitaria. Este modelo es una evolución en los modelos educativos de educación a distancia. Tanto es así que hoy, a diferencia de lo que sucedió con los modelos de educación a distancia anteriores basados en la televisión, la radio o la video conferencia, este modelo asíncrono esta siendo usado también por muchas universidades en todo el mundo, de forma complementaria a las clases presenciales. Es lo que hemos convenido en llamar modelo híbrido de formación.

El modelo educativo de la UOC, centrado en el estudiante y basado en la asincronía, no es un modelo de autoaprendizaje. Como ya hemos comentado el papel del profesor, en cualquiera de sus cuatro modalidades mencionadas, es determinante. El estudiante se relaciona con el consultor y con el tutor, de la misma forma que lo haría en una clase presencial, con la diferencia que esta relación se lleva a cabo de forma asíncrona.

\section{La formación del profesorado para la docencia online}

Desde la creación de la UOC, ésta ha significado para las demás universidades un sistema alternativo de formación de su profesorado en el uso de las tecnologías en la docencia. Todos los profesores que actúan como consultores en la UOC deben 
superar un programa breve en formación online. Esto ha significado que, a partir de la existencia de la UOC, el profesorado universitario de Cataluña ha podido formarse y ejercitarse como formador online. Sin duda la UOC ha sido una plataforma de formación privilegiada para muchos profesores y profesionales en el uso docente de Internet.

Conseguir que un profesor universitario asuma como propia toda esta metodología docente es otra de las funciones que tiene el profesor de la UOC. El profesor de la UOC se ha convertido en un formador de profesores de docencia virtual, ya que la formación y experiencia que recibe el consultor de la UOC es utilizada por estos profesores en su universidad de origen de todo el entorno universitario español.

\section{La apuesta por las innovaciones tecnológicas}

Cuando la UOC inició su primer curso académico no existían las plataformas tecnológicas educativas actuales. Fue necesario invertir en la creación de una plataforma nueva, específica para la UOC, llamada Campus Virtual. Hoy, once años después, la realidad es otra. Muchas universidades, no sólo en Cataluña, han aprovechado la experiencia tecnológica de la UOC para el desarrollo de plataformas educativas en Internet o para el desarrollo de módulos administrativos complementarios para la gestión académica universitaria. Actualmente el reto es la migración de las plataformas educativas actuales hacia formatos en software libre, a la vez que se abren los contenidos educativos en la red. Una vez más la UOC, juntamente con las otras universidades catalanas y financiada por el Gobierno Catalán, está liderando un proyecto adaptación de los entornos tecnológicos para el aprendizaje a formatos de software libre.

Las nuevas apuestas tecnológicas de la UOC pasan ahora por el trabajo de las comunidades de software abierto (Open Source) y por la dinámica de los recursos educativos en abierto (Open Educational Resources). La rectora de la UOC, Dra. Imma Tubella, firmó a inicios de 2006 la Declaración de Berlín que aboga por el uso de Software Libre y Recursos Educativos en Abierto ${ }^{12}$.

\section{La cooperación en investigación}

Desde su inicio, la UOC quiso estar presente en proyectos de investigación. En primer lugar, y durante los primeros años, estos proyectos fueron de investigación aplicada, basados en convocatorias europeas, especialmente en el marco del desarrollo tecnológico y de educación y nuevas tecnologías. Estos proyectos se llevaron a cabo 
juntamente con otras universidades catalanas y europeas. Posteriormente la UOC, con una clara voluntad de convertirse en una universidad de investigación, inició sus programas de investigación básica, centrados en el estudio de las transformaciones en la Sociedad de la Información. La investigación, por tanto, se centra principalmente en el análisis de los cambios que se producen en cada una de las disciplinas en las que trabaja la UOC, en el marco de la Sociedad de la Información y del Conocimiento.

\section{La Cátedra UNESCO de e-learning}

La Cátedra UNESCO de e-learning de la UOC, creada el 2 de noviembre de 2002, es el resultado también de una apuesta institucional por la innovación educativa y tecnológica continuada de la UOC. Los objetivos reflejados en el convenio entre la UNESCO y la UOC muestran la intención mutua de construcción de un espacio internacional de reflexión, análisis y cooperación sobre la superación de las desigualdades en educación superior a partir del uso de las Tecnologías de la Información y de la Comunicación. Con este fin la Cátedra UNESCO de e-learning se posiciona como un espacio de integración de la innovación tecnológica y educativa, no sólo para la UOC si no también para muchas instituciones iberoamericanas que cooperan y participan en ella.

La Cátedra UNESCO de e-learning se configura a partir de los siguientes pilares de actividad y de reflexión:

- Análisis de la situación de la Educación Superior en la Sociedad del Conocimiento

La Cátedra UNESCO de e-learning de la UOC ha llevado a cabo diferentes seminarios internacionales con la finalidad de analizar la situación de cambio de las instituciones de educación superior producida por las transformaciones en la sociedad a partir de las tecnologías de la información y de la comunicación. Estos seminarios se han dirigido a rectores, vicerrectores y directivos de instituciones de educación superior con la finalidad de poder incidir directamente en las estrategias y políticas de cada institución.

- Difusión de estudios y análisis sobre la relación entre la Universidad y la Sociedad del Conocimiento

La aportación más relevante de la Cátedra UNESCO de e-learning a la difusión es la creación de la Revista de Universidad y Sociedad del Conocimiento, RUSC, (www.uoc.edu/rusc) en el año 2004. RUSC es una revista electrónica semestral, indexada, que trabaja a partir de la revisión entre pares de un 
comité científico y acogida a la licencia abierta de Creative Commons ${ }^{13}$. Cada número se compone de un monográfico de alto nivel académico que analiza diferentes ámbitos de la universidad en la sociedad de la información.

Aparte de la revista la Cátedra UNESCO de e-learning ha trabajado conjuntamente con la Biblioteca Virtual de la UOC en la creación de un centro de documentación electrónica que abarca los diferentes elementos educativos, tecnológicos y organizativos del e-learning. También distribuye un boletín electrónico de información académica sobre e-learning cada semana, en el que se destacan tres artículos académicos sobre el tema, aparecidos en revistas indexadas y una web informativa o de recursos sobre educación y tecnología.

- Cooperación internacional en la Sociedad Red

Siendo la cooperación uno de los ejes fundamentales establecidos en el convenio de creación de la Cátedra UNESCO de e-learning ésta ha procurado desde el inicio incorporar esta visión y acción en sus actividades. Se ha trabajado priorizando las acciones con universidades e instituciones de educación superior de América Latina, trabajando en algunos casos conjuntamente con la AECI (Agencia Española de Cooperación Internacional) y la ACCD (Agència Catalana per a la Cooperació i el Desenvolupament).

Aparte del énfasis en la cooperación de todas las actividades de la Cátedra (en los seminarios y en las actividades de difusión aparecen siempre elementos que estimulan la cooperación) queremos destacar la creación de la RIU (Red de Innovación Universitaria) el mes de diciembre de 2006 en Lima, Perú, con la participación de más de 30 universidades iberoamericanas. Esta Red es el resultado de años de trabajo de cooperación interuniversitaria en el marco de la Cátedra UNESCO de E-learning.

La RIU centrará sus actividades en diferentes nodos temáticos, con énfasis en elementos de innovación, tales como: Materiales Educativos en Abierto, Plataformas de Software Libre, compartir y crear cursos en común, acreditación de la calidad de programas online, etc. Tiene también la voluntad de influir directamente en las políticas educativas de los países latinoamericanos a través de la acción de los rectores de las universidades participantes en la red. 


\section{LA UOC ANTE LOS RETOS ACTUALES}

La UOC, como universidad de la Sociedad de la Información, debe estar constantemente abierta a la incorporación de innovaciones y activa para observar e interpretar los retos que la sociedad demanda. A finales del 2005, después de diez años de su creación, la UOC cambio por primera vez de rector pasando a ser, en palabra de la nueva rectora Dra. Imma Tubella, "de un gran proyecto a una gran universidad."

Para continuar liderando el ámbito de la innovación en el uso educativo de las TIC en educación superior, la UOC necesita estar en un constante proceso de revisión y renovación interno. En este sentido algunos de los elementos ya expuestos como el proceso de abertura del Campus Virtual y de los contenidos, o los procesos de multilingüismo, por citar algunos, son un ejemplo de ello. Seguidamente vamos a exponer algunos de los retos clave, a nuestro entender, en un futuro inmediato para esta universidad. Retos que ya se dibujan y ante los cuales la organización esta ya habilitando propuestas y acciones concretas.

\section{Innovación en educación y en tecnología}

Además del camino recorrido ya en el campo de la innovación en la UOC, los retos de futuro hoy se enmarcan en saber interpretar adecuadamente la relación, cada vez más intensa, entre evolución tecnológica y educativa en el marco de la educación superior. Es cierto que la evolución tecnológica ha marcado en estos diez últimos años el camino de la innovación educativa, y sin duda parece que va a continuar haciéndolo. Pero también es cierto que desde los ámbitos educativos -y también tecnológicos- se ha ido situando la tecnología en su adecuado lugar de instrumento básico de apoyo a la realización de los procesos de enseñanza y aprendizaje y a la consecución de los objetivos de aprendizaje en entornos sociales diversos como los actuales.

En las universidades presenciales, por ejemplo, se ha seguido un camino rápido desde la conectividad a la portabilidad, pasando por la movilidad en cuanto a la concepción de cómo debían ser los sistemas de conexión de los estudiantes y profesores a las TIC a partir de los dispositivos tecnológicos (Duart; Lupiáñez, 2005). En otras palabras, se ha pasado de las aulas de informática a los puntos de conexión en las aulas y en los pasillos, a ceder y alquilar o prestar ordenadores portátiles a los alumnos y a dotar los Campus presenciales de conexión inalámbrica abierta. La UOC está avanzando en la utilización de nuevos dispositivos de conexión, como los 
teléfonos portátiles, PDA, etc. Existe un proyecto innovador denominado My way ${ }^{14}$ que incorpora en diferentes dispositivos y en diferentes formatos los materiales de aprendizaje de la UOC.

El potencial de lo que hemos convenido en llamar Web 2.0 es muy elevado para el mundo educativo. El paso de un sistema web de acceso a una información con un nivel de participación un tanto bajo a un nuevo sistema en el que la interacción y la construcción de conocimiento compartido es la base es, sin duda, una buena noticia para el mundo educativo. Herramientas como los wiki, dinámicas como las que proporcionan los Podcast, páginas de información y participación como los Blogs, o páginas de intercambio como el deli.co.ous, por citar algunos ejemplos, son muy interesantes para generar dinámicas educativas en educación superior. La UOC está participando ya en proyectos de innovación educativa en los que se analiza el potencial de estas herramientas.

Pero más allá del uso de estas herramientas o dispositivos en educación, las transformaciones se centran en aquellas que incorporan ya un nivel de reflexión y de filosofía de comportamiento, como es el movimiento por el software libre o ahora ya el de los Contenidos Educativos en Abierto (Open Educational Resources). La UOC, por iniciativa del Consejo de Gobierno, y a partir de las acciones de la Cátedra UNESCO de e-learning, está trabajando activamente para incorporar esta filosofía en su actuación. El paso de un Campus Virtual propietario a uno en código abierto, trabajando para ello conjuntamente y de forma cooperativa con todas las universidades catalanas es un paso decidido en este sentido. Pero también lo es la apuesta por la apertura de los materiales de aprendizaje que ya se ha realizado en el Master en Software Libre, por ejemplo ${ }^{15}$. Al igual que MIT, Rice University, o de forma especial la Open University del Reino Unido que ya han decidido abrir parte de sus contenidos la UOC se encuentra en un proceso similar que culminará en un período no excesivo de tiempo. Sin duda esta apuesta no debe ser considerada únicamente como una decisión tecnológica; es claramente una apuesta por la calidad y por la abertura a la sociedad de la dinámica y conocimiento universitario (Wiley, 2007).

\section{Calidad en la formación, acreditación del profesorado y de los servicios académicos}

La UOC ha demostrado ya su apuesta por la calidad en la formación. El nivel de sus egresados y la satisfacción de los mismos lo avalan. Los reconocimientos internacionales así lo muestran también. El sello de oro de reconocimiento de 
calidad otorgado por EFQM (European Fundation for Quality Management) o la reciente evaluación de la EUA (European University Asociation) así lo muestran. Pero además se plantea como reto dar un paso adelante que se focaliza en tres niveles de concreción: el análisis de la calidad de la formación que se ofrece -comprende los diferentes agentes y objetos que forman parte del proceso educativo y de los programas-; la acreditación del profesorado de la UOC; y la acreditación de los servicios académicos que se ofrecen.

La calidad de la formación se articula en la validación o acreditación externa de los programas que la UOC ofrece. De entrada debemos destacar que la UOC ha contribuido decididamente en la comprensión y facilitación, por parte de las agencias evaluadoras de la Calidad (AQU en Cataluña, ANECA en España, y otras a nivel internacional) de los niveles diferenciales para el análisis de la calidad entre los programas presenciales y los ofrecidos de forma online o virtual. La UOC ha participado en la determinación de los indicadores de calidad para la evaluación de entornos virtuales de aprendizaje y de materiales de aprendizaje online. Ahora, además, está siendo evaluada en sus programas al igual que el resto de universidades, aunque como es lógico, con unos parámetros propios adecuados para las instituciones de educación superior que actúan en la virtualidad. El reto hoy es acreditar los programas de la UOC especialmente en el marco de las directrices del Espacio Europeo de Educación Superior (EEES). Se han iniciado ya evaluaciones de programas de Grado y de Postgrado y se avanza hacia la acreditación paulatina de la totalidad de los programas.

El profesorado de la UOC tiene unas características propias y específicas, que ya hemos expuesto anteriormente. La acreditación del mismo es fundamental para equipararlo al resto de profesorado universitario y establecer mecanismos internos y externos de evaluación y mejora. El profesorado doctor de la UOC acaba de superar ampliamente el proceso de acreditación de la Agencia Catalana de Calidad Universitaria (AQU) que evalúa la formación, docencia e investigación realizada por el profesorado. El reto es continuar en esta dinámica y fortalecer aquellos profesores de nueva incorporación para superar estos objetivos.

De igual forma la evaluación de los servicios en una institución no presencial es muy importante. Nos referimos a servicios administrativos o de documentación como la Biblioteca Virtual. En educación virtual superior la percepción de calidad por parte de los estudiantes no sólo proviene de los niveles de atención por parte de los profesores o de los elementos o personas que intervienen en el proceso de aprendizaje. La percepción de calidad está mediatizada por los servicios complementarios 
como por ejemplo: que el Campus Virtual funcione las 24 horas del día, que la Secretaria Académica responda las dudas que se plantean, que los cobros se efectúen adecuadamente o que la biblioteca informe y atienda en la solicitud de información que se le demanda. Dos acciones que se estan desarrollando muestran la apuesta por trabajar en estos retos. Por una parte los servicios de administración académica y de atención al estudiante están trabajando conjuntamente con sus homólogos de la Open University del Reino Unido analizando los elementos necesarios de mejora y aprendiendo de la experiencia mutua. $\mathrm{O}$ en otro nivel debemos destacar la reciente evaluación que la Agencia Catalana de Calidad Universitaria (AQU) ha realizado satisfactoriamente a la Biblioteca Virtual de la UOC.

\section{Internacionalización de los programas y multilingüismo}

Internacionalizar los programas no es sólo un reto para cualquier institución de educación superior, es además una necesidad. En la Sociedad del Conocimiento en la que nos encontramos no podemos pensar en realizar los programas formativos de forma aislada. El trabajo en común y la cooperación interuniversitaria es una necesidad que aumentan los niveles de competitividad y excelencia de los programas. En Europa el llamado proceso de Bolonia establece claros requerimientos en este sentido.

La UOC desde sus inicios ha realizado una apuesta clara por la internacionalización de su acción y de sus programas. Las aulas de la UOC, especialmente en los cursos de postgrado que tienen unos requisitos de acceso menos restrictivos por normativa estatal, tienen unos buenos niveles de internacionalización. Pero no sólo es este el nivel que interesa actualmente. El reto consiste en el trabajo en común con instituciones de educación superior para la elaboración conjunta de programas e impartición de los mismos. La UOC está enfatizando su relación con universidades europeas para la construcción conjunta de programas.

En la creación de programas conjuntos con universidades europeas o americanas influyen algunos elementos que debemos tener en cuenta. En primer lugar la evolución de las tecnologías que ya hemos comentado facilita bastante esta apuesta de cooperación. Ya no es necesario compartir un mismo tipo de Campus Virtual, o tener los materiales de formación en el mismo soporte tecnológico. Las tecnologías de código abierto y la estandarización de los formatos va a favor de este tipo de iniciativas. Pero además, la aceptación por parte de las universidades presenciales de la virtualidad como un elemento de complementariedad de la formación también 
ha facilitado este tipo de alianzas. Existen pocas universidades presenciales que no dispongan ya de espacios de virtualidad, y a su vez existe también más flexibilidad en las instituciones virtuales para poder realizar actos presenciales debido a que los mismos pueden ser inmediatamente situados en la red y por ello accesibles de forma asíncrona por parte de cualquier estudiante. El reto, por tanto, es ahora más evidente: ser capaz de construir relaciones fructíferas y académicas de calidad en beneficio de la consecución de programas con alto nivel de rigor académico y metodológico.

Como exponíamos anteriormente la UOC a través de la Cátedra UNESCO de elearning está impulsando la Red de Innovación Universitaria (RIU). Esta Red será el marco de desarrollo para este tipo de relaciones, no sólo por parte de la UOC sino por parte de cualquiera de las universidades que de ella forman o formen parte en un futuro. La Red esta pensada que funcione en nodos, al igual que las redes de la Sociedad de la Información y del Conocimiento, y sólo se pide a sus participantes que sean capaces de aportar a la construcción de la misma, no como espectadores sino como participantes activos. Esta Red, hoy por hoy esta constituida por universidades del ámbito iberoamericano, por tanto con tan sólo dos lenguas comunes de trabajo, el español y el portugués, que ya tiene en su haber un buen nivel de cooperación. Pero no se descarta en absoluto la incorporación en la Red de universidades europeas o de América del Norte con lo que el inglés u otras lenguas podrían estar presentes en breve.

Por ello y por la dinámica de la UOC en su relación europea el multilingüísmo es un elemento clave en esta política de internacionalización. Ya hoy en la UOC es fácil celebrar reuniones o seminarios plenamente en inglés, algo que no sucedía en sus primeros años de historia. Sin ir más lejos, las actividades que realiza la Cátedra UNESCO de e-learning, están adoptando el idioma inglés como base. El III Seminario Internacional ${ }^{16}$ se celebró ya en esa lengua, sin traducción simultánea, y las Rountables internas que se organizan con profesores invitados y profesores propios son también en esa lengua. Sin duda, se trata de un esfuerzo complementario nada habitual en países de habla hispana pero que asumimos como un reto para la internacionalización y la mejora de nuestros programas.

Pero no es sólo el inglés la lengua con la que se está trabajando. El nuevo vicerrectorado en Multilingüismo que la rectora ha creado trabaja en la confección de entornos virtuales en lengua francesa, portuguesa e incluso árabe. $Y$ disponemos ya de entornos en chino mandarín y en japonés para nuestros programas de estudios asiáticos. Estamos, sin duda, ante un reto muy interesante y necesario ante el que las universidades, por nuestra propia misión, no podemos estar ajenos. 
Todo esta apuesta por la internacionalización y el multilinguismo en los programas formativos de la UOC tendrá su articulación básica en los programas de postgrado. Los programas de grado lo serán también en gran medida, pero los de postgrado y doctorado, por su especificad temática y diversidad de aproximación serán sin duda -como ya lo son ahora- las plataformas más importantes en este ámbito de cooperación interuniversitaria internacional. En este sentido la UOC se encuentra ante el reto de definir su política de postgrado, algo que dirimirá en breve apostando por fortalecer el prestigio académico de sus cursos y su internacionalización.

\section{Investigación centrada en la Sociedad de la Información}

La UOC es una universidad joven, pero a pesar de ello apostó tempranamente por no ser tan sólo una universidad centrada en la docencia (teaching university) sino también una universidad de investigación. Este reto, asumido ya hace un tiempo con la creación e impulso al IN3 (Internet Interdisciplinary Institute) está avanzando de forma clara y coherente con los demás retos expuestos que relacionan la calidad de la formación, el profesorado y la internacionalización de los programas.

La Comisión Científica del IN3 presidida por el Profesor Manuel Castells agrupa expertos internacionales que aportan su visión y experiencia en el desarrollo de la acción de investigación en la UOC. Sus informes apuestan por intensificar la acción investigadora centrándola en el análisis de los diferentes ámbitos de conocimiento con los que trabaja la UOC relacionados con la Sociedad de la Información.

Recientemente se han constituido alrededor de treinta grupos de investigación ${ }^{17}$ en el seno del IN3, dirigidos por profesores doctores de la UOC y en los que participan profesores también de otras universidades españolas y europeas. Los estudiantes de doctorado y becarios de investigación se sitúan en estos grupos y realizan sus tesis doctorales en los mismos. El reto está ahora en avanzar en la consecución de investigaciones doctorales o no en el seno de estos grupos.

De igual forma el trabajo en el análisis de la calidad de la investigación es determinante. Si bien pareciera que en este campo existe ya un claro sistema evaluativo (publicaciones, tesis, revistas, proyectos, etc.) el IN3 va a trabajar también en la definición de un sistema de evaluación de la investigación que contemple la realidad actual de la difusión del conocimiento a través de la web. La misma web del $\mathrm{IN}_{3}$-renovada en breve en este sentido- así como la indexación de las revistas académicas de la UOC apuesta por establecer esta nueva dinámica. 


\section{Universidad y Empresa}

También desde sus inicios la UOC ha tenido como objetivo la relación como universidad con el mundo empresarial. Inicialmente la misma universidad, organizada como Fundación sin afán de lucro, creó conjuntamente con diferentes empresas otras con la finalidad de satisfacer sus propias necesidades, favoreciendo así la creación de una organización más flexible. Por ejemplo, la creación de la empresa Eureka Media $^{18}$ conjuntamente con otra empresa del sector editorial favorece la producción de los contenidos de aprendizaje propios de la UOC, así como su difusión conjunta con la editorial creada por la propia UOC que recibe el nombre de EdiUOC $^{19}$. Pero la finalidad inicial no fue únicamente servir a los intereses de producción de la propia universidad sino que se crearon empresas como Gestión del Conocimiento (GEC) ${ }^{20}$ destinadas a la difusión del saber hacer (know how) adquirido por la UOC en la formación virtual al mundo de la empresa, especialmente en la formación de los recursos humanos o en la creación de lo que se ha convenido en llamar universidades corporativas.

El nuevo equipo rector de la UOC ha aportado también como un reto específico el de la relación de la universidad con el mundo empresarial, no sólo a partir de la creación de empresas (spin off) como las citadas, sino a partir de la acción del desarrollo del emprendedurismo entre los estudiantes, los convenios con las empresas $^{21}$ y la transferencia del conocimiento o investigación. El objetivo es el trabajo sistemático en la idea de la UOC como universidad emprendedora (Clark, 1998).

Qué duda cabe que, por su especificidad virtual, la UOC no tiene este camino de relación con el mundo empresarial tan sencillo como lo pueda tener una universidad politécnica por ejemplo. Pero en tanto que universidad de la Sociedad de la Información en un ámbito nuevo que es el de la Economía del Conocimiento (Torrent, 2002) parece que sí puede y debe encontrar su camino de relación. De esta forma se está actuando a través de construir la relación con las empresas a partir de las aportaciones en el conocimiento, o trabajar directamente con empresas que en si mismas ya son catalogadas como empresas del conocimiento. Un caso interesante es el del Centro de Excelencia en e-learning ${ }^{22}$, creado con SUN Microsystems, que tiene un nivel internacional relevante.

Una línea interesante de crecimiento y de cooperación con el mundo empresarial, aparte de la ya citada del fomento del espíritu emprendedor de los alumnos de la UOC, es la de la elaboración de estrategias de formación conjuntas encaminada a la 
mejora de los equipos de las empresas asociadas o no a la UOC. Esta estrategia que se relaciona con la de Postgrado de la UOC aumenta el nivel de profesionalización de los programas y satisface las necesidades concretas formativas de grandes sectores profesionales.

\section{NOTAS}

$1 \quad$ http://www.uoc.edu/web/esp/universidad/indexhome.html

2 Normas de Organización y Funcionamiento de la Universitat Oberta de Catalunya

3 http://www10.gencat.net/dursi/es/un/sist universitats cat.htm

4 Ley 3/1995, de 6 de abril, de reconocimiento de la UOC, publicada en el DOGC $\mathrm{n}^{0} .2040$ de $21 / 04 / 95$.

5 Ley 3/2006, de 17 de març, de creació del Fons d'Acció Territorial de l'Educació Superior. DOGC núm. 4604 - 30/03/2006 Con esta ley se establece un fondo de acción territorial para financiar aquellos centros de educación superior que tengan un fuerte impacto en la dinamización del territorio o en la innovación empresarial de un determinado sector empresarial con relevancia estratégica territorial. Se trata, de una clara y reciente medida de descentralización de la política universitaria catalana.

6 http://www.uoc.edu/web/eng/university/popup/model educatiu.html

7 En el estado español además del castellano (español) existen otras tres lenguas oficiales en algunas regiones concretas: catalán, gallego y vasco. El catalán es lengua oficial en Cataluña, Valencia e Islas Baleares.

8 Otro ejemplo de investigación aplicada es la que se lleva a cabo en relación a los contenidos educativos abiertos, el software libre o el derecho a la educación en el estado del Bienestar en la Sociedad del Conocimiento. Líneas de investigación que hallan en la propia institución su primera razón de ser, y por lo tanto la financiación y la aplicación.

$9 \quad$ El proceso de creación del Espacio Europeo de Educación Superior ha puesto el acento en algunos aspectos estructurales como son la redefinición de los planes de estudio, la implantación de los créditos European Credit Transfer System, las agencias de garantía de la calidad, el suplemento europeo de título, la evaluación, las competencias, etc. Sin embargo, el espíritu que ha impulsado todas estas transformaciones es la creación de un espacio europeo de educación superior que debe ser un espacio guiado por los valores europeos, esto es, un espacio social y cultural y para ello, hay que sensibilizar a los estudiantes de la realidad de Europa como un espacio social, democrático, plurilingüe y multicultural.

10 http://www.uoc.edu/in3/esp/ 
11 Cada semestre los profesores propios de la UOC de los resultados de las encuestas realizadas a los estudiantes sobre su grado de satisfacción sobre los recursos docentes, sobre el planteamiento de la evaluación y sobre la acción docente.

12 http://oa.mpg.de/openaccess-berlin/berlindeclaration.html

13 www.creativecommons.org

14 http://www.uoc.edu/in3/myway/

15 http://www.uoc.edu/masters/oficiales/master oficial software libre/master oficial software libre materiales.htm

16 http://www.uoc.edu/catedra/unesco/seminario/english/

17 http://www.uoc.edu/in3/eng/grups recerca.html

18 http://www.eurecamedia.com/

19 http://www.editorialuoc.com/

20 http://www.gec.es

21 http://www.uoc.edu/web/esp/empresas/empresas aso.html

22 http://www.uoc.edu/premsa/?p=433

\section{REFERENCIAS BIBLIOGRÁFICAS}

Cabero, J. (2006). Bases pedagógicas del e-learning. Revista de Universidad y Sociedad del Conocimiento, 3(1).

Clark, B. R. (1998). Creating Enterpreneurial Universities. Oxford: IAU Press Pergammon.

Delgado, A. M.; Oliver, R.; Salomón, L. (2006). Hacia un aula virtual plurilingüe y multicultural. Revista de Educación a Distancia (15).

Dochy, F.; Segers, M.; Dierick, S. (2003). Nuevas vías de aprendizaje y enseñanza y sus consecuencias: una nueva era en la evaluación. $R E D-U, 2(18)$.

Duart, J. M.; Kiselyova, E. (2003). The Open Universtiy of Catalonia: A Local-Global University Model, in: Varis, T.; Usumi, T.; Klemm, W. (Eds.) Global Peace Through The Global University System. Tampere: GUS.

Duart, J. M.; Lupiáñez, F. (2005). E-strategias en la introducción y uso de las TIC en la universidad. Revista de Universidad y Sociedad del Conocimiento RUSC, 2. [en línea]. Disponible en: www.uoc.edu/ rusc/dt/esp/duart0405.pdf. [Consulta: 2006, 26 de noviembre]

Duart, J. M.; Sangrà, A. (2000). Aprender en la virtualidad. Barcelona: Gedisa.

Onrubia, J. (2005). Aprender y enseñar en entornos virtuales: actividad conjunta, ayuda pedagógica y construcción del conocimiento. Revista de Educación a Distancia (numero monográfico II).

Tiffin, J.; Rajasingham, L. (2003). The Global Virtual University. London: Routledge Falmer.

Torrent, J. (2002). Innovació tecnològica, creixement econòmic $i$ economia del coneixement: una aproximació empírica, agragada $i$ internacional a la incroporació del coneixement a l'activitat productiva durant la dècada dels noranta. Barcelona: Universitat Oberta de Catalunya.

Wiley, D. (2007). Open content is incredibly powerful advertising for universities. Món: UOC. 


\section{PERFIL ACADÉMICO Y PROFESIONAL DE LOS AUTORES}

Lourdes Salomón Sancho. Es profesora de Derecho Romano de los Estudios de Derecho y Ciencia Política de la Universidad Oberta de Catalunya. Doctora en Derecho por la Universidad Rovira i Virgili de Tarragona (1999) y ha cursado un Postgrado en Derecho de la Contratación Electrónica UOC-Illes Balears (2005).

Sus líneas de investigación son el Derecho Romano y las nuevas metodologías docentes aplicadas al aprendizaje superior.

Ha realizado estancias de investigación en las universidades de Göttingen, Salzburg y Tübingen.

E-mail:1salomon@uoc.edu

Pablo Lara Navarra. Licenciado en Documentación y Diplomado en Biblioteconomía y Documentación por la Universidad de Granada. Dirige y colabora en proyectos de investigación sobre e-learning, redes sociales y organización y recuperación de información centrada en el área de gestión de información, conocimiento y contenidos. Participa en diferentes forums y cursos especializados en administración electrónica, recuperación de información en entornos electrónicos, capacitación docente e institucional en la organización y gestión en e-learning.

E-mail:plara@uoc.edu

Josep M. Duart, Doctor en Pedagogía por la Universidad Ramon Llull y Master in Business Administration (MBA) por ESADE Business School. Actualmente es profesor en el máster de e-Learning de la UOC y del programa de doctorado sobre la Sociedad de la Información y el Conocimiento, área e-Learning, de la UOC.

Ha participado en la elaboración del modelo pedagógico de la UOC y ha asesorado como consultor experto en la aplicación de metodologías de formación en línea a diferentes universidades de España y Latinoamérica.

Como investigador del Internet Interdisciplinary Institute (IN3) de la UOC y miembro del grupo de investigación Education and Network Society (ENS) dirige y colabora en proyectos de investigación sobre $e$-Learning centrados en organizaciones educativas y uso del -Learning. Es coordinador de la Cátedra UNESCO de e-Learning de la UOC y director académico del Seminario Internacional Liderar la Universidad en la Sociedad del Conocimiento. Además, es director de la Revista de Universidad y Sociedad del Conocimiento.

E-mail: jduart@uoc.edu 


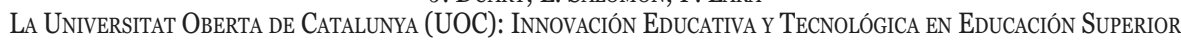

DIRECCIÓN DE LOS AUTORES

Universidad Oberta de Cataluña

Avda. Tibidabo 39

Barcelona, 08035. España

Fecha de recepción del artículo: 22/01/07

Fecha de aceptación del artículo: 20/03/07 